\title{
NMR Study on the Dissolved State of Polyacrylonitrile in Various Solvents
}

\author{
Makiko Hattori, Hitoshi Yamazaki, Masatoshi Saito, Kunio Hisatani, \\ and Kunihiko OKaJima \\ Fundamental Research Laboratory of Natural \& Synthetic Polymers, Asahi Chemical Industry Co., Ltd., \\ 11-7 Hacchonawate-cho, Takatsuki, Osaka 569, Japan
}

(Received December 15, 1995)

\begin{abstract}
An attempt was made to elucidate the dissolved state of polyacrylonitrile (PAN) in various solvents by analyzing ${ }^{13} \mathrm{C}$ NMR chemical shift difference for the arbitrarily chosen two NMR peaks derived from PAN molecules and by considering solvent effect on the electro-magnetical parameters introduced previously with aid of solvation phenomena of PAN in solvents. For this purpose, R-PAN and $\gamma$-PAN employed hithetofore were subject to the analysis. Solvents employed included typical organic solvents such as dimethylformamide (DMF), dimethylacetamide (DMAc), dimethyl sulfoxide (DMSO), and aqueous (aq) inorganic solvents such as $70 \mathrm{wt} \%$ aq nitric acid $\left(\mathrm{HNO}_{3}\right), 55 \mathrm{wt} \%$ aq sodium thiocyanate (NaSCN). The dissolved state or the solvation state of PAN in organic solvent was mainly discussed in comparison with that in aq $\mathrm{HNO}_{3}$, which is known most powerful solvent even for highly isotactic PAN. It was found that interaction of all solvents to polymer is strictly dependent on polymer stereoregularity and the solvent power is related to the solvent ability to interact on the long meso $(m)$ sequence part in PAN. Aq $\mathrm{HNO}_{3}$ shows quite different interaction form with nitrile group in $m m$ and $m r(r$, racemo) sequences rather without forming $\pi$ electron conjugate system. In contrast to this, the organic solvents proved to have individually favorable interaction sites more or less forming $\pi$ electron conjugate system with nitrile group in polymer. The favorable polymer site was $m m r$ for DMSO and $m r$ for DMF and DMAc. Solvation number of solvent was proved to be in good accordance with that theoretical solvation number, calculated by assuming that each solvent solvates on its favorable sites in polymer. The tentative solvation form for each solvent is proposed.

KEY WORDS Nuclear Magnetic Resonance / Polyacrylonitrile / Solvation / Dimethyl Sulfoxide / Dimethylformamide / Dimethylacetamide c / $\pi$ Electron Conjugate System /
\end{abstract}

Atactic polyacrylonitrile (PAN) is well known to dissolve into concentrated inorganic salt solution (for example, sodium thiocyanate ( $\mathrm{NaSCN})$ ), concentrated inorganic acid solvents (aq nitric acid $\left(\mathrm{HNO}_{3}\right)$, sulfuric acid), and polar organic solvents such as $N, N^{\prime}$-dimethylformamide (DMF), $N, N^{\prime}$-dimethylacetamide (DMAc), dimethyl sulfoxide (DMSO), and ethylene carbonate (EC). The concentrated solutions of PAN in these solvents have been extensively employed in the commercial production of acrylic fibers. ${ }^{1}$ It is very true that structure and properties of commercial acrylic fibers differ from another to one another. Although we should bear in mind that the commercial acrylic fibers are not made from PAN homopolymer but from different copolymer types of PAN, one of important factors controlling the final properties and structures of acrylic fibers might be attributable to the difference in the solvent employed as far as the wet-spinning method is concerned. Recently, Kamide and his coworkers ${ }^{2-5}$ carried out a very detailed solution study on atactic PAN (R-PAN), synthesized by redox polymerization and highly isotactic PAN ( $\gamma$-PAN), polymerized by $\gamma$-ray irradiation on acrylonitrile (AN)-urea canal complex, by light scattering, solution viscosity, and solvation technique. They revealed that I) PAN having isotactic triad content larger than $50 \%$ is not easily dissolved in DMF, but is readily soluble in $67 \mathrm{wt} \%$ aq nitric acid, II) conformation parameter for $\gamma$-PAN in solvent is far larger than that for R-PAN, leading to a conclusion that there exist some ladder-like polymer chain part in $\gamma$ PAN. In another line of study on PAN, they ${ }^{6,7}$ have also proposed a ladder-like chain for meso $(m)$ sequence part of which neighboring nitrile $(-\mathrm{C} \equiv \mathrm{N})$ groups interact with each other to bring about the double bond nature, and later its possibility was confirmed by quantum chemical calculation. ${ }^{8}$ On the one hand, Kamide et al. $^{9}$ reported that cellulose solutions obtained by dissolving cellulose with different crystal forms into a same solvent ( $9 \mathrm{wt} \%$ aq sodium hydroxide or cadoxen) exhibited quite different flow birefringence phenomena. Authors ${ }^{10}$ also have pointed out that cellulose acetates dissolved in different polar solvents showed the specific solvation form depending on solvent employed. The different dissolved state of poly-( $\alpha$-methylstyrene $)$ in benzene or cyclohexane, which is known as relatively nonpolar solvent, have been also pointed out by Kamide et al. ${ }^{11}$ The study on the dissolved state of polyamide (nylon 66) in calcium chloride/methanol mixture (calcium chloride fraction in the mixture is denoted as $W_{\mathrm{ca}}$ ), carried out also by authors, ${ }^{12}$ has revealed that nylon 66 chain takes very compact conformation in the solvent mixture with $W_{\mathrm{ca}}=0.1-0.15$, and in the range of $W_{\text {ca }} \geqq 0.15$ the dissolution of nylon 66 takes place by direct interaction of the very bulky and meta-stable association form of solvent mixture to polymer. These facts suggest that the mechanism of dissolution process (and probably the dissolved state) would be dependent of solvent as well as inherent nature of polymer and significantly influence the properties of the resultant polymer solutions.

The dissolved state of PAN in various solvents has not yet fully understood at molecular level, although up to present time several attempts ${ }^{13-16}$ have been made: Kobayasi ${ }^{13}$ observed a colloid particle with negative electric charge obtained by adding water into PAN/DMF solution and speculated without clear evidence the following polymer solvent interactions shown in (a) and (b). 


$$
\begin{gathered}
\text { OI } \\
\left(\mathrm{CH}_{3}\right)_{2}-\mathrm{N}-\mathrm{C}-\mathrm{H} \cdots \mathrm{N}=\mathrm{C}- \\
\left(\mathrm{CH}_{3}\right)_{2}-\mathrm{N}-\underset{\mathrm{C}}{\mathrm{C}}=\mathrm{O} \cdot \mathrm{H}-\mathrm{C}-\mathrm{CN} \\
\mathrm{H}
\end{gathered}
$$

Suzuki ${ }^{14}$ studied the interaction of DMF and isobutyronitrile (IBN) as model of PAN by infrared (IR) and Raman spectroscopy and revealed the strong polarizability of amide group in DMF such as $-\mathrm{N}^{+}=\mathrm{C}-\mathrm{O}^{-}$ and the non-ionic nature of aldehyde hydrogen in DMF, concluding that there is a possibility of hydrogen bond between such polar part of DMF with $\alpha$-methine hydrogen in IBN as shown in (b) but not in (a). Suzuki ${ }^{15}$ also suggested the interaction between $-\mathrm{C} \equiv \mathrm{N}$ group in IBN and amide group in DMF. Recently, Yamazaki ${ }^{16}$ has studied in more detail the solvent (DMF, DMSO, aq $\mathrm{HNO}_{3}$ ) interaction on IBN by ${ }^{1} \mathrm{H},{ }^{13} \mathrm{C},{ }^{15} \mathrm{~N}$ NMR, IR, and Raman spectroscopies. He reported that 1) the interaction of $-\mathrm{C} \equiv \mathrm{N}$ in IBN with DMSO is stronger than with DMF, 2) methyl proton in DMSO might participate in the interaction with $-\mathrm{C} \equiv \mathrm{N}$ in IBN, 3) the interaction of DMSO with methine proton in IBN is much weaker than that with DMF, 4) non-dissociated form of aq $\mathrm{HNO}_{3}$ (that is, highly concentrated aq solution) facilitates the dissolution of PAN, 5) the interaction of aq $\mathrm{HNO}_{3}$ with IBN resulted in the shift of the stretching vibration of $-\mathrm{C} \equiv \mathrm{N}$ to higher wave number side, which is a reverse effect as in the case for DMF and DMSO, and 6) $\mathrm{N}-\mathrm{OH}$ in aq $\mathrm{HNO}_{3}$ might interact with $-\mathrm{C} \equiv \mathrm{N}$ of in IBN rather than $\mathrm{O}=\mathrm{N}=\mathrm{O}$ part in $\mathrm{HNO}_{3}$, as shown in (c).

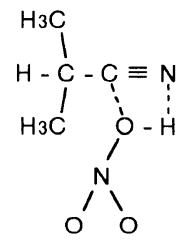

These results deduced using model compound are basically important but unfortunately applicable only to acrylonitrile (AN) monomer units in racemo $(r)$ arrangement in the PAN molecular chain and doubtlessly showed only that $-\mathrm{C} \equiv \mathrm{N}$ and $-\mathrm{CH}$ group in PAN play predominantly important role in its dissolution to solvent, being far from the detailed understanding of the dissolved state of PAN. Because PAN is a stereoregular polymer whose $m$ configuration includes internal interaction between neighboring two AN units ${ }^{6,7}$ and in addition solvents generally used for PAN (for examples, DMF, DMSO, DMAc, aq $\mathrm{HNO}_{3}$ and aq NaSCN) all have double or triple bonds (or dipolar nature) in their molecules having molecular sizes larger than AN unit, then such circumstances should be taken into consideration in discussing the dissolved state of PAN in solvents. As an example, it is plausible to consider that a solvent molecule can interact with two or three AN units. So far, authors have introduced several electro-magnetical parameters for ${ }^{13} \mathrm{C}$ NMR peak assignment of PAN and AN/vinylidene chloride copolymer based on pentad tacticity of AN units. ${ }^{6,17}$ However, the parameters in the above studies were introduced only for describing the interaction of the neighboring $\mathrm{AN}$ units in polymer, ignoring solvent effect. It has been found that ${ }^{13} \mathrm{C}$ NMR peaks of PAN in different solvent appeared at somewhat different chemical shift and the chemical shift difference between arbitrarily chosen two peaks also differ depending on solvent used. Thus, the detail analysis on ${ }^{13} \mathrm{C}$ NMR peaks of PAN in different solvents might give some insight for explaining the solvent-polymer (monomer unit) interaction, hence, the dissolved state of PAN in solvent.

In this study we attempt to elucidate the dissolve state of PAN in various solvents as an extension of the previous study ${ }^{6}$ by taking the so-called solvent nature into the above consideration with help of the recent advance in NMR technique, which seems to allow us to challenge again the above unsolved problem. In this connection, one another important factor, the solvation phenomena is also considered.

\section{EXPERIMENTAL}

\section{Polymer Samples}

Two $\gamma$-PAN whole polymer samples differing in $\mathrm{mm}$ triad fraction (coded as $\gamma$-PAN-1, $m m=0.52 ; \gamma$-PAN-2, $m m=0.59$ ) and a R-PAN (coded as R-PAN, $m m=0.26$ ), prepared in the previous paper, ${ }^{1}$ were utilized. Pentad tacticity data and the sequence probability $(P(m / r)=$ $0.518, P(r / m)=0.197$ for $\gamma$-PAN-1 and $P m=0.519$ for R-PAN) were also cited from the same literature. All sample was subject to sound velocity measurement and only R-PAN sample was subject to NMR measurement.

\section{Solvents}

Reagent grade DMF, DMSO, DMAc, and $70 \mathrm{wt} \%$ aq $\mathrm{HNO}_{3}$, supplied by Kishida Chemicals Co., Osaka, were used as received for sound velocity measurement of PAN solution. Deuterated dimethylformamide (DMF$d_{7}$ ), DMSO- $d_{6}$, DMAc- $d_{9}, 70 \mathrm{wt} \% \mathrm{DNO}_{3}$, and $\mathrm{D}_{2} \mathrm{O}$ (all supplied from Merck, West Germany) were used for ${ }^{13} \mathrm{C}$ NMR measurements. A guaranteed grade $\mathrm{NaSCN}$, supplied Kishida Chemicals Co., was diluted by $\mathrm{D}_{2} \mathrm{O}$ to give $55 \mathrm{wt} \%$ solution and used for NMR measurement.

\section{Solvation}

The adiabatic compressibility of the PAN solutions was calculated from the sound velocity and density of the solutions and converted to the mole number of the solvated solvent per 1 monomer unit of polymer $\mathrm{Nm}$ using Passynsky's relation. ${ }^{18}$ The sound velocity was measured on a Nusonic Model 6080 Concentration Analyzer at $25 \pm 0.005^{\circ} \mathrm{C}$ in the polymer concentration range of $1-5 \mathrm{~g}$ polymer $/ 100 \mathrm{~g}$ solvent. The details of the measuring conditions have been described already. ${ }^{5}$

\section{NMR Measurement}

${ }^{13} \mathrm{C}$ NMR measurements on PAN/solvent systems were made on a FT-NMR spectrometer model JEOL FX-400 (100.7 MHz for ${ }^{13} \mathrm{C}$ nucleus) at $70^{\circ} \mathrm{C}$ for $\mathrm{R}-$ PAN solutions dissolved in DMF- $d_{7}$, DMSO- $d_{6}$, DMAc$d_{9}$, and $\mathrm{NaSCN} / \mathrm{D}_{2} \mathrm{O}$, and at $30^{\circ} \mathrm{C}$ for R-PAN $/ 70 \mathrm{wt} \%$ $\mathrm{DNO}_{3}$ solution. DSS and dioxane were used as standard for $\mathrm{NaSCN} / \mathrm{D}_{2} \mathrm{O}$ and $70 \mathrm{wt} \% \mathrm{DNO}_{3}$, respectively. TMS was used as standard for all other solvent. Measuring conditions are as follows: $45^{\circ}$ pulse, width $10 \mu \mathrm{s}$, 
repetition $10 \mathrm{~s}$, data point $16 \mathrm{~K}$, number of pulse $64 \times 67$.

\section{RESULTS AND DISCUSSION}

Table I collects the solvation number of typical solvents (DMSO, DMF, DMAc, $\mathrm{HNO}_{3}$ ) on PAN samples. The dissolving power of these solvents judging from the solvation number for R-PAN was the order of $70 \mathrm{wt} \%$ aq $\mathrm{HNO}_{3} \gg \mathrm{DMAc}=\mathrm{DMF}>\mathrm{DMSO}$, but for highly isotactic PAN this order was $70 \mathrm{wt} \%$ aq $\mathrm{HNO}_{3} \gg \mathrm{DMSO}$ $\mathrm{DMAc}=\mathrm{DMF}$. This clearly shows that not only species of solvent but also the stereoregularity of PAN is one of factors to control the solvent power. The mole number of the solvated solvent/ $1 \mathrm{~mol}$ of $\mathrm{AN}$ unit is estimated to be $0.5-0.56$ for aq $70 \mathrm{wt} \% \mathrm{HNO}_{3}$, being larger for PAN polymer with higher $\mathrm{mm}$ fraction. For DMSO the value of $c a$. 0.21 is evaluated irrespective of polymer stereoregularity. However, the solvation number of DMF and DMAc, which have weaker dissolving power toward higher stereoregular PAN than DMSO, was estimated as $0.30-0.32$ against R-PAN. These facts suggest that the stereoregularity of PAN has an effect on the site factor of solvation. The solvation number of DMF and DMAc for $\gamma$-PAN failed to be measured due to non-dissolution of the PAN sample. These results indicate that the solvent power is not decided from only the solvation number and the solvation of solvent to PAN molecule should be dependent of its stereoregularity. Therefore, it is interesting to clarify the dissolved state of PAN in view of the solvation of solvent and the stereoregularity of PAN. The interaction between PAN molecule and solvent might give a definite electrical and magnetical circumstances for carbon nuclei constituting $\mathrm{AN}$ unit $\left(-\mathrm{CN},-\mathrm{CH}\right.$, and $\left.\mathrm{CH}_{2}\right)$, depending on solvent nature (that is, different solvation pattern or different interaction form), as was proved in our previous study on polymer-solvent interaction using IBN as model for PAN. ${ }^{16}$ Therefore, analysis on ${ }^{13} \mathrm{C}$ NMR spectra of PAN/solvent systems may give some information on the dissolved state (polymer-solvent interaction) of PAN in given solvents. As Kamide et $a l .{ }^{6}$ proposed previously, the experimentally observed chemical shifts for $-\mathrm{CN}$ and $-\mathrm{CH}$ carbons are products of parameters $\left(\delta, \sigma_{1}, \sigma_{2}, \varepsilon\right.$ for $-\mathrm{CN}$ carbon; $-\omega, \delta^{\prime},-\varepsilon^{\prime}$ for- $\mathrm{CH}$ carbon), and these parameters have been proved to the relative order of chemical shifts of carbons due to pentad tacticity of PAN. Note that all these parameters are introduced only for describing the interactions between monomer units constituting a PAN molecules. Therefore, if PAN is dissolved without any interaction with solvent the magnitude of these parameters should all be constant, irrespective of species of solvent. Since

Table I. Number of solvent solvated on monomer units of PAN

\begin{tabular}{lccccc}
\hline & & \multicolumn{4}{c}{ Solvation number $\left(\mathrm{mol} \mathrm{mol}^{-1}\right)$} \\
\cline { 3 - 6 } $\begin{array}{c}\text { Polymer } \\
\text { sample }\end{array}$ & $\begin{array}{c}\text { Triad } \\
\text { isotacticity }\end{array}$ & \multicolumn{4}{c}{ Solvent } \\
& & DMF & DMSO & DMAc & $\mathrm{aq} \mathrm{NHO}_{3}$ \\
\hline R-PAN & 0.26 & 0.32 & 0.21 & 0.30 & 0.51 \\
$\gamma$-PAN-1 & 0.52 & - & 0.22 & - & 0.52 \\
$\gamma$-PAN-2 & 0.59 & - & - & - & 0.56 \\
\hline
\end{tabular}

these parameters were first introduced to express the intrinsic nature of polymer itself without considering solvent effect, then the difference in the apparent values for these parameters can be regarded as the results of specific interaction between PAN molecule and the solvent in question. In turn, the specific interaction can be predicted by considering electrical and magnetical influences on PAN molecule derived from the specific interaction satisfying the apparently observed values of these parameters. The followings are review of these parameters:

$\delta ; \quad$ an apparent disappearance effect of the shielding effect existed on isolated $\mathrm{CN}$ carbon of the central monomer unit in $r r$ arrangement, induced by double bond nature in a $m$ arrangement (applicable to the central $\mathrm{CN}$ carbon in $r r \cdot m r$, $m r \cdot m r, r r \cdot m m, ~ m r \cdot m m, r m \cdot m r, r m \cdot m m$, and $\mathrm{mm} \cdot \mathrm{mm}$ arrangements; $\cdot$ means central).

$\sigma_{1}$; an electron density decreasing effect on central $\mathrm{CN}$ carbon in $r \cdot m$ and $m \cdot m$ arrangement (. means central; for the latter $2 \sigma_{1}$ is given).

$\sigma_{2} ;$ an electron density decreasing effect on central $\mathrm{CN}$ carbon in ${ }^{*} m m$ (* means central).

$\varepsilon ; \quad$ a deshielding effect on central $\mathrm{CN}$ carbon arranged in $r$ configuration by induced magnetical field generated with circular current on the double bond nature of the adjacent $m$ configuration $(* r m ; *$ means central).

$\delta^{\prime} ; \quad$ an apparent disappearance effect of the shielding effect existed on isolated $\mathrm{CH}$ carbon of the central monomer unit in $r r$ arrangement, induced by double bond nature in a $m$ arrangement (applicable to the central $\mathrm{CH}$ carbon in $r \cdot m$ and $m \cdot m$ arrangements; $\cdot$ means central).

$-\omega$; an apparent electron density increasing effect on $\mathrm{CH}$ carbon based on the decrease in electronegativity of $\mathrm{CN}$ group in the same monomer caused by double bond nature arranged in $m$ configuration (applicable to $r \cdot m$ and $m \cdot m$, - means central; for the latter $-2 \omega$ is given).

$-\varepsilon^{\prime}$; a deshielding effect on central $\mathrm{CH}$ carbon arranged in $r$ configuration by induced magnetical field generated with circular current on the double bond nature of the adjacent $m$ configuration (applicable to $r \cdot m$ and $m \cdot m$, ' means central; for the latter $-2 \varepsilon^{\prime}$ is given).

Note that $-\mathrm{C} \equiv \mathrm{N}$ carbon NMR peak is separable based on pentad tacticity and $\mathrm{CH}$ carbon peak is separable only based on triad tacticity. The magnitude of these parameters can be estimated as the difference in chemical shifts between arbitrarily chosen two NMR peaks. Table II collects some of these parameters evaluated experimentally from NMR spectra of PAN/solvents systems (Solvent; DMF, DMSO, DMAc, $70 \mathrm{wt} \% \mathrm{HNO}_{3}$, and as a reference data for $55 \mathrm{wt} \% \mathrm{NaSCN}$ ) and also includes the chemical shift difference between center $\mathrm{CH}$ (or $\mathrm{CN}$ ) peak in $m r$ arrangement and center $\mathrm{CH}_{2}$ peak $(\Delta \mathrm{CH}$, $\Delta \mathrm{CN})$. Numbers in parenthesis denote the order of the magnitude for given parameters among solvents except for aq NaSCN of which solvent structure itself is not clear at present. Therefore, the discussion concerning aq $\mathrm{NaSCN}$ will be made in only limited case. From the table the most characteristic feature is seen for aq $\mathrm{HNO}_{3}$ : 
Table II. Electro-magnetical parameters of R-PAN in various solvents, evaluated by ${ }^{13} \mathrm{C}$ NMR spectra (parameters or peak difference, expressed in ppm)

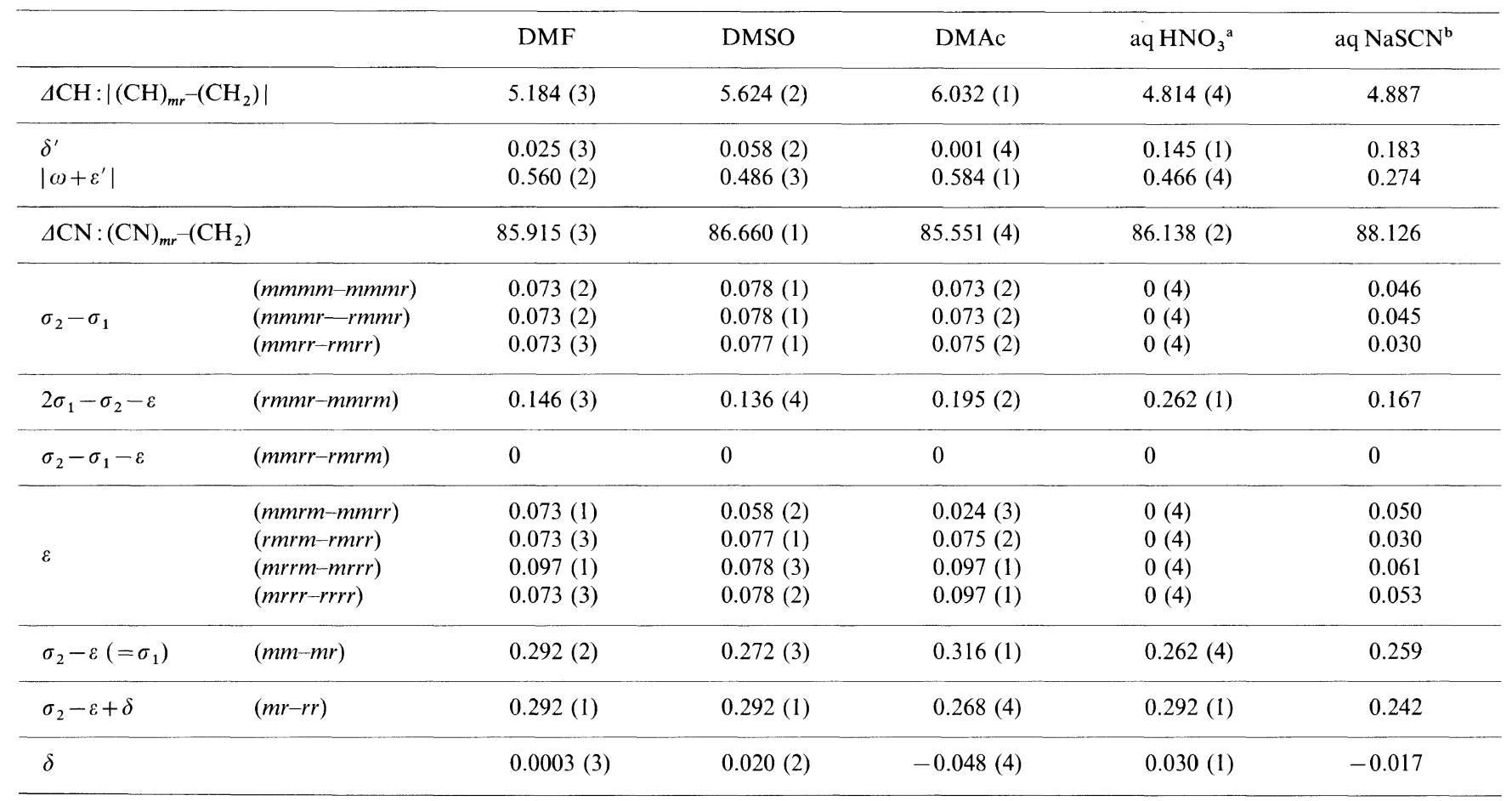

${ }^{\text {a,b }}$ Measured from dioxane and sodium 2,2-dimethyl-2-silapentane-5-sulfonate (DSS), respectively (for others; tetramethylsilane (TMS)).

$\sigma_{2}=\sigma_{1}(=0.262)$ and $\varepsilon=0$. This means that aq $\mathrm{HNO}_{3}$ coordinates with or stays on non-selectively $\mathrm{mm}$ and $m r$ sequences within the time scale of NMR measurement but not with or on $r r$ sequence so as to keep $\sigma_{1}$ effect on $\mathrm{CN}$ carbon inherent to polymer stereoregularity. The magnitude of $\sigma_{1}$ working on $-\mathrm{C} \equiv \mathrm{N}$ carbon in polymer reflects the conjugated double bond nature by $\pi$ electron, and the larger $\sigma_{1}$ denotes the formation of stronger $\pi$ electron conjugate system with solvent. The $\sigma_{1}$ value is lowest for aq $\mathrm{HNO}_{3}$, suggesting no inclusion of $\mathrm{O}=\mathrm{N}=\mathrm{O}$ part in $\mathrm{HNO}_{3}$ in the conjugate interaction with $-\mathrm{C} \equiv \mathrm{N}$, as has been pointed out by Yamazaki. ${ }^{16}$ The disappearance of $\varepsilon$ effect might be resulted by shielding effect from the coordinated or solvated aq $\mathrm{HNO}_{3} . \Delta \mathrm{CH}$ is a direct measure for shielding effect on $\mathrm{CH}_{2}$ carbon in question brought about by double bond nature of $-\mathrm{C} \equiv \mathrm{N}$ and/or polar site of solvent since the direct interaction of solvent to $\mathrm{CH}_{2}$ in polymer, which induces the change in electron density on $\mathrm{CH}_{2}$ carbon, is not anticipated. The rough estimation of the shielding effect from the interacted solvent can be made using the value $\Delta \mathrm{CH}-\delta^{\prime}$ because $\delta^{\prime}$ reflects the degree of deviation from triple bond in $-\mathrm{C} \equiv \mathrm{N}$. The order of the magnitude of $\Delta \mathrm{CH}-\delta^{\prime}$ was found to be the same to $\Delta \mathrm{CH}$. Then, larger the $\Delta \mathrm{CH}$, higher the shielding effect and is lowest for a aq $\mathrm{HNO}_{3}$. This means that $\mathrm{O}=\mathrm{N}=\mathrm{O}$ part in $\mathrm{HNO}_{3}$, which is a possible shielding source, should be located to give relatively low shielding effect on $\mathrm{CH}_{2}$ of monomer unit in a polymer-solvent interaction form. The $\delta$ value evaluated is a measure for direct interaction and strength of interaction of solvent on $-\mathrm{C} \equiv \mathrm{N}$ to inspire more double bond nature in $-\mathrm{C} \equiv \mathrm{N}$ or to interact with $-\mathrm{C} \equiv \mathrm{N}$ so as to exclude the shielding effect on $-\mathrm{C} \equiv \mathrm{N}$ from the triple bond, being highest $(\delta=0.030)$ for aq $\mathrm{HNO}_{3}$. This inevitably leads us to a conclusion that
H-O-N- part in $\mathrm{HNO}_{3}$ is a definite interaction site with $-\mathrm{C} \equiv \mathrm{N}$. The above effect should be parallel to $\delta^{\prime}$ effect on $\mathrm{CH}$ carbon from the definition, which is again highest for aq $\mathrm{HNO}_{3}$. If there is non-specific interaction between polymer and solvent, the larger $\delta^{\prime}$ must result in larger $\omega+\varepsilon^{\prime}$. If this does not realize we should consider some specific interaction between polymer and solvent. The effect of $\delta^{\prime}$ and $\omega$ works anti-parallel way on $\mathrm{CH}$ by their definitions. Then, in the case where such interaction takes place the value $\left(\omega+\varepsilon^{\prime}\right)-\delta^{\prime}$ might be a measure for shielding effect from the coordinated solvent molecule and direct interaction of solvent to $\mathrm{CH}$ proton. To roughly estimate the degree of the direct interaction on $\mathrm{CH}$, the value $\left(\omega+\varepsilon^{\prime}\right)-\delta^{\prime}-\Delta \mathrm{CH}$ can be useful because the shielding effect on $\mathrm{CH}$ and $\mathrm{CH}_{2}$ within a monomer unit is of roughly the same order. The order of the magnitude of $\left(\omega+\varepsilon^{\prime}\right)-\delta^{\prime}-\Delta \mathrm{CH}$ among solvents was found to be the same as the value $\left(\omega+\varepsilon^{\prime}\right)$, then the value $\left(\omega+\varepsilon^{\prime}\right)$ are used as representative for $\left(\omega+\varepsilon^{\prime}\right)-\delta^{\prime}$ and $\left(\omega+\varepsilon^{\prime}\right)-\delta^{\prime}-\Delta \mathrm{CH}$. From the literatures, ${ }^{13-16}$ the direct interaction of solvent to $\mathrm{CH}$ proton in PAN might occur by taking lone pair electron in solvent, leading to an increase in electron density on $\mathrm{CH}$ carbon. Therefore, the larger $\left(\omega+\varepsilon^{\prime}\right)$ value corresponds to the larger degree of direct interaction and the value is lowest for aq $\mathrm{HNO}_{3}$. Keeping the above discussion in mind the result shown in Table II are summarized as follows: (1) The order of $\Delta \mathrm{CH}$ or $\Delta \mathrm{CH}-\delta^{\prime}$ is DMAc > DMSO $\gg \mathrm{DMF}>\mathrm{HNO}_{3}$, hence the degree of the shielding effect from the interacted solvent (double bond part) is also in the above order, (2) the larger is the apparent $\delta^{\prime}$ effect on $\mathrm{CH}$ carbon centered at $m r$ triad sequence, unexpectedly the smaller is the $\omega+\varepsilon^{\prime}$ effect, indicating the specific interaction between polymer and solvents, that is, this fact implies that $-\mathrm{CN}$ loses a nature 
of triple bond but does'nt make the $\pi$ electron conjugate system, (3) the order of magnitude of $\delta^{\prime}$ is similar to $\delta$ and being $\mathrm{HNO}_{3} \gg \mathrm{DMSO}>\mathrm{DMF} \gg \mathrm{DMAc}$, suggesting that the strength of interaction of solvent to $-\mathrm{C} \equiv$ $\mathrm{N}$ is in the above order, (4) the order of magnitude of $\omega+\varepsilon^{\prime}$, which is a measure for the degree of direct interaction to $-\mathrm{CH}$ proton in polymer, is DMAc $>\mathrm{DMF} \gg$ DMSO $>\mathrm{HNO}_{3}$, (5) $\sigma_{2}-\sigma_{1}$ evaluated by three methods is constant in most solvents (DMSO $(=0.078 \mathrm{ppm})>$ $\mathrm{DMF}=\mathrm{DMAc}(=0.073 \mathrm{ppm}) \gg \mathrm{HNO}_{3}(0 \mathrm{ppm})(\mathrm{NaSCN}$ $(0.046-0.030 \mathrm{ppm})$ is positioned just between DMAc and $\left.\mathrm{HNO}_{3}\right)$ ), (6) for all solvents $\sigma_{2}-\sigma_{1}=\varepsilon$ holds (therefore, $\varepsilon=0 \mathrm{ppm}$ for $\mathrm{HNO}_{3}$ ) when evaluated from the chemical shift difference between mmrr and rmrm peaks, and interestingly for organic solvents the evaluated $\sigma_{2}-\sigma_{1}(=\varepsilon)$ values fall almost on $0.073-0.078$, (7) some exceptions appear among the $\varepsilon$ values evaluated by four other methods depending on solvent nature (DMF, $\varepsilon(=0.097 \mathrm{ppm})$ evaluated from the chemical shift difference between $\mathrm{mrrm}$ and $\mathrm{mrr}$; DMSO, $\varepsilon(=0.058$ ppm) evaluated from mmrm-mmrr); DMAc (0.024, $0.097 \mathrm{ppm}$ ) evaluated from $m m r m-m m r r$ and $m r r m-m r r$ (or mrrr-rrrr), (8) apparent $\sigma_{1}$ effect, which reflects the easiness of formation of $\pi$ electron conjugate system between solvent and $-\mathrm{C} \equiv \mathrm{N}$, is in the order of DMAc $(0.316 \mathrm{ppm})>\mathrm{DMF} \quad(=0.292 \mathrm{ppm})>\mathrm{DMSO} \quad(=0.272$ ppm) $>\mathrm{HNO}_{3} \quad\left(=0.262 \mathrm{ppm}\right.$ ) (for $\mathrm{NaSCN} \sigma_{1}=0.259$ ppm), (9) solvent dependence of apparent $\delta$ effect on $\mathrm{CN}$ carbon in PAN is in the order of $\mathrm{HNO}_{3}(=$ $0.03 \mathrm{ppm})>\mathrm{DMSO}(=0.02 \mathrm{ppm})>\mathrm{DMF}(=0.00 \mathrm{ppm})$ $\gg$ DMAc $(=-0.48 \mathrm{ppm})$, showing characteristically a negative value for DMAc.

Facts (5), (8), and (9) indicate that the interaction of $\mathrm{HNO}_{3}$ with $-\mathrm{C} \equiv \mathrm{N}$ in $m m$ and $m r$ sequence of polymer is quite different from that for other organic solvents, which basically form $\pi$ electron conjugate system with $-\mathrm{C} \equiv \mathrm{N}$ more or less, and might correspond to the facts reported by Yamazaki ${ }^{16}$ (see, 4) and 5) in the present text). Facts (3) and (4) seem to correlate with so-called solvent power against highly isotactic PAN. Fact (4) for DMF and DMSO is comparable to the prediction deducted by Yamazaki ${ }^{16}$ that the direct interaction of DMSO to $-\mathrm{CH}$ in polymer is much weaker than that of DMF. This means that $-\mathrm{CH} \leftarrow \mathrm{O}=\mathrm{S}$-type interaction is restricted for DMSO but the interaction type shown in (b) is possible for DMF. Fact (5) indicates that interaction of organic solvents to $-\mathrm{C} \equiv \mathrm{N}$ on long meso sequence site in polymer is regarded as negligible except for DMSO, since DMF and DMAc do not dissolve the highly isotactic PAN. This is important feature in dissolving PAN with ladder-like structure (long meso sequence site) by attacking such part and leading to an increase in entropy of displacement of polymer chain in solvent. This well corresponds to $\sigma_{2}=\sigma_{1}$ evaluated for aq $\mathrm{HNO}_{3}$, denoting that meso in long $m$ sequence behaves as an isolated meso by the result of solvent interaction. More detailed inspection shows that DMAc tends to slightly interact with $-\mathrm{C} \equiv \mathrm{N}$ on $m m r$ or $r m r$ sequence. Fact (7) clearly suggests that each solvent has individually favorable interaction site in polymer so as to give different $\varepsilon$ values. For DMSO, mmrm or $m m r r$ (hence, probably $m m r)$ site is favorable, comparable to the explanation given for Fact (5). The $m r r$ site is most favorable for
DMF and the $m m r$ and $m r r$ (hence, $m r$ ) sites are for DMAc. These results indicate that the solvent interaction to polymer strongly depends on stereoregularity of polymer and the $-\mathrm{C} \equiv \mathrm{N}$ on the terminal end monomer unit in $m$ or longer $m$ sequence of polymer always participates in the interaction with solvent. This is reasonable because $-\mathrm{C} \equiv \mathrm{N}$ on the terminal end monomer unit in $m$ or longer $m$ sequence is most ionized in nature. The necessity of $\mathrm{mm}$ sequence site in polymer for interaction with DMSO is characteristic, meaning that much more ionization of $-\mathrm{C} \equiv \mathrm{N}$, which is realized only for terminal end in long $m$ sequence, is required for interaction with DMSO. Fact (8) gives an interesting conclusion that the order of magnitude of $\sigma_{1}$ is just in parallel with dipole moment of solvents. The negative $\delta$ value for DMAc shown in Fact (9) should be considered in some details: From Facts (1), (4), and (9) DMAc must interact with both $-\mathrm{C} \equiv \mathrm{N}$ and $-\mathrm{CH}$ in a monomer unit in question so that the shielding effect from solvent works on $\mathrm{CH}_{2}$ group in the same monomer unit, and on the basis of the above interaction the $-\mathrm{C} \equiv \mathrm{N}$ carbon must also be placed in a shielding area from double bond nature in solvent. This situation might occur when amide group of DMAc takes the form $-{ }^{\delta+} \mathrm{N}=\mathrm{C}=\mathrm{O}^{\delta-}$, or $-\mathrm{N}^{+}=\mathrm{C}-\mathrm{O}^{-}$as pointed out for DMF by Suzuki. ${ }^{14}$ The second lowest value of $\delta(=0.00)$ for $\mathrm{DMF}$ is also explained from the above point of view. From the above discussion and consideration of magnetical influence caused by interacted solvent we tentatively show the polymer-solvent interactions, which are dependent of stereoregularity of PAN, in Figure 1. In all interactions one mole of solvent interact with 2 or $3 \mathrm{AN}$ monomer units in the individually favorable site $(\mathrm{mm}$ and $\mathrm{mr}$ for $\mathrm{HNO}_{3}, m r r$ or $m r$ for DMF and DMAc, $m m r$ for DMSO) and naturally one end of polymer site capable of interaction with solvent is $-\mathrm{C} \equiv \mathrm{N}$ in a monomer unit located at the terminal end of $m$ sequence.

Since the above interactions are drawn from the NMR analysis on polymer side dissolved in solvent, then it is needed for solvent molecule to be strongly trapped with several polar sites in polymer within a time scale of NMR measurement. Therefore, the interaction might closely correlate with solvation phenomena, shown in Table I. Assuming that each solvent stays on its favorable site (triad or tetrad) of polymer but use 2 or 3 AN units, then theoretically trapped solvent on polymer $\left(\mathrm{mol} \mathrm{mol}^{-1}\right)$ can be calculated and the results are shown in Table III. Here, the base pentad fraction is extended to septad sequence fraction by using the reaction probabilities and the solvent in question is allocated to its favorable site on the septad sequence by assuming that an $\mathrm{AN}$ monomer unit used for one interaction with solvent cannot participate in another solvent interaction. For aq $\mathrm{HNO}_{3}$ the theoretical solvation numbers on $m m$ and $m r$ for $\gamma$-PAN-1 and R-PAN are 0.49 and 0.51 , respectively, being in excellent agreement with those determined experimentally $(0.51$ and 0.52 , respectively). Since the experimental solvation number for higher isotactic PAN of which $\mathrm{mm}$ fraction becomes large, increases to 0.56 , the solvation of aq $\mathrm{HNO}_{3}$ on $\mathrm{mm}$ and $\mathrm{mr}$ triads is reasonable. For DMSO the theoretical solvation number on $m m r$ for $\gamma$-PAN-1 and R-PAN are estimated to be $c a$. 0.15 and 0.17 , re- 

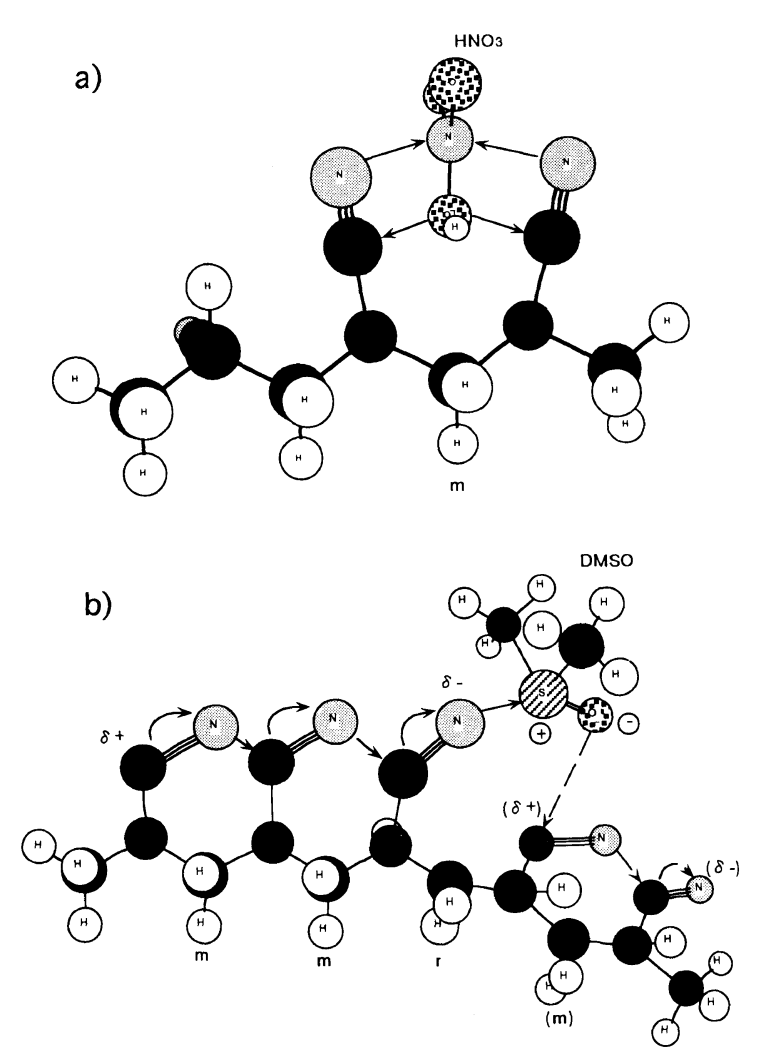
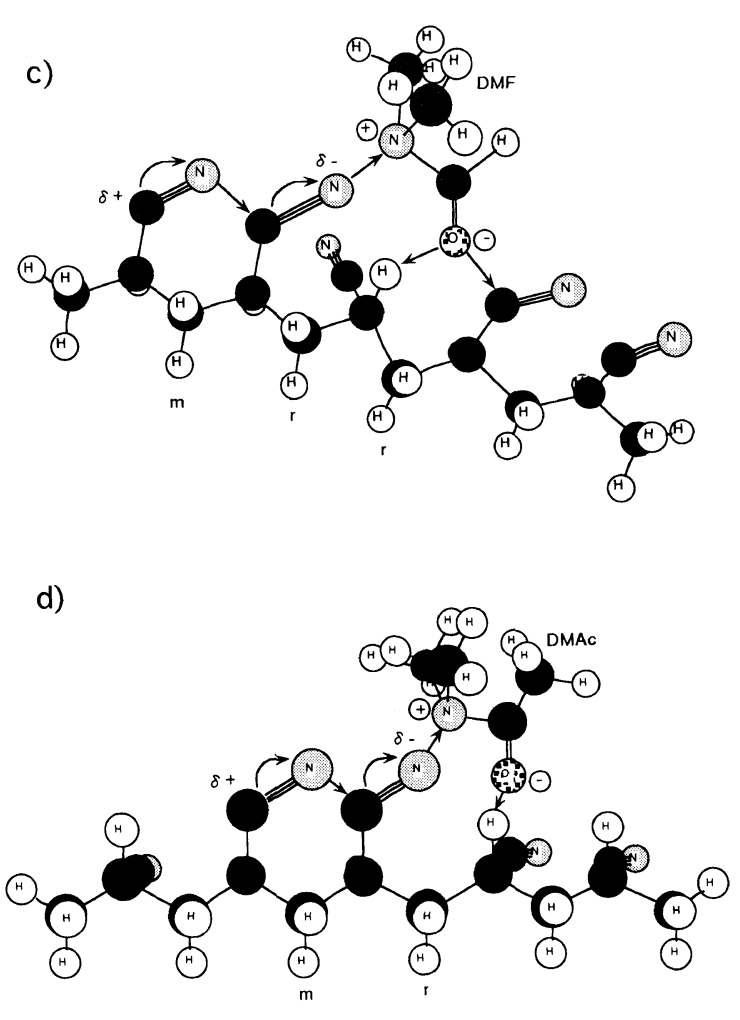

Figure 1. Schematic presentation of PAN-solvent interactions. a) PAN-aq $\mathrm{HNO}_{3}$, b) PAN-DMSO; -.- means a possible interaction when the adjacent AN unit is arranged in $m$; c) PAN-DMF; d) PAN-DMAc.

Table III. Caluculated number of solvent solvated on monomer units of PAN $(\mathrm{mol} / \mathrm{mol})^{\mathrm{a}}$

\begin{tabular}{|c|c|c|c|c|c|c|c|c|c|c|c|c|}
\hline \multirow{3}{*}{$\begin{array}{c}\text { Base } \\
\text { pentad }\end{array}$} & \multirow{2}{*}{\multicolumn{2}{|c|}{$\begin{array}{c}\text { Pentad fraction } \\
\text { (Obsd) }\end{array}$}} & \multicolumn{10}{|c|}{ Solvent solvation $(\mathrm{Calcd}) \times 10^{-2} \mathrm{~mol} \mathrm{~mol}^{-1}$} \\
\hline & & & \multicolumn{2}{|c|}{$m r$ coordina. } & \multicolumn{2}{|c|}{$m m$ coordina. } & \multicolumn{2}{|c|}{$m m r$ coordina. } & \multicolumn{2}{|c|}{$m r r$ coordina. } & \multicolumn{2}{|c|}{$m r m$ coordina. } \\
\hline & $\gamma$-PAN & R-PAN & $\gamma$-PAN & R-PAN & $\gamma$-PAN & R-PAN & $\gamma$-PAN & R-PAN & $\gamma$-PAN & R-PAN & $\gamma$-PAN & R-PAN \\
\hline $\mathrm{mmmm}$ & 0.374 & 0.077 & 2.456 & 1.234 & 0.174 & 0.032 & $2.456^{\mathrm{b}}$ & $1.234^{\mathrm{b}}$ & 1.014 & 0.508 & 1.090 & 0.549 \\
\hline$m m m r$ & 0.115 & 0.125 & 2.294 & 3.085 & 0.046 & 0.050 & $2.294^{b}$ & $3.085^{\mathrm{b}}$ & 0.533 & 1.415 & 1.008 & 1.526 \\
\hline$r m m r$ & 0.040 & 0.068 & 1.600 & 2.720 & 0.008 & 0.014 & 1.600 & 2.720 & 0.262 & 1.090 & 0.690 & 1.176 \\
\hline $\mathrm{mmrm}$ & 0.118 & 0.125 & 2.742 & 4.079 & 0.036 & 0.034 & 2.345 & 3.080 & 0.320 & 0.822 & 2.029 & 2.677 \\
\hline$m m r r$ & 0.073 & 0.097 & 2.086 & 3.233 & 0.022 & 0.026 & 1.575 & 2.079 & 1.141 & 1.705 & 0.010 & 0.346 \\
\hline $\mathrm{rmrm}$ & 0.087 & 0.149 & 3.480 & 5.960 & 0.012 & 0.013 & 1.160 & 1.288 & 0.815 & 2.144 & 2.417 & 4.328 \\
\hline$r m r r$ & 0.055 & 0.129 & 1.368 & 3.265 & 0 & 0 & 0.653 & 0.993 & 1.357 & 3.183 & 0.917 & 2.149 \\
\hline$m r r m$ & 0.029 & 0.055 & 0.674 & 1.795 & 0.004 & 0.047 & 1.472 & 0.952 & 0.453 & 0.966 & 0.846 & 0.392 \\
\hline$m r r r$ & 0.051 & 0.112 & 0.881 & 1.937 & 0.007 & 0.009 & 0.798 & 1.400 & 1.100 & 2.401 & 0.074 & 0.399 \\
\hline$r r r$ & 0.057 & 0.065 & 0.984 & 1.124 & 0 & 0 & 0.258 & 0.500 & 0.984 & 1.124 & 0 & 0 \\
\hline Total & 1.00 & 1.00 & 18.56 & 28.43 & 30.95 & 22.48 & $\begin{array}{c}14.62 \\
\left(19.37^{b}\right)\end{array}$ & $\begin{array}{c}17.33 \\
\left(21.65^{\mathrm{b}}\right)\end{array}$ & 7.98 & 15.36 & 9.08 & 13.54 \\
\hline
\end{tabular}

${ }^{\mathrm{a}}$ Fractions of hexad, septad are calculated by using following probability parameters: For $\gamma, P(r / m)=0.197, P(m / r)=0.518$; for R-PAN, $P m=0.519$.

${ }^{\mathrm{b}}$ Calculated by assuming that solvent-solvent associate (not isolated solvent) is solvated on polymer).

spectively. The values are almost the same but is slightly lower than those ( 0.22 and 0.21 , respectively) experimentally observed. Since DMSO requires more ionized $-\mathrm{C} \equiv \mathrm{N}$ to interact with polymer, compared with other organic solvents, if we assume that $-\mathrm{C} \equiv \mathrm{N}$ in terminal monomer unit on longer $m$ sequence (at least $\mathrm{mmm}$ ) is strongly ionized and can accommodate the solvent-solvent associate beside isolate solvent, then, the solvation numbers for $\gamma$-PAN-1 and R-PAN fall on the same value $(0.20,0.22$, respectively), which is very close to those evaluated experimentally. DMF and DMAc might be essentially $m r$ coordination. The theoretical solvation number for R-PAN is $c a$. 0.28 , which is very close to the observed value $(0.30-0.32)$, but that theoretical one for $\gamma$-PAN-1 is 0.19 , which might be insufficient to dissolve $\gamma$-PAN. Thus, the solvation phenomena observed for PAN/solvent systems could be reasonably explained by the dependence of solvent interaction on polymer stereoregularity, reduced from ${ }^{13} \mathrm{C}$ NMR analysis on polymer side dissolved in solvents.

\section{CONCLUSIONS}

Analyzing ${ }^{13} \mathrm{C}$ NMR chemical shift difference and 
the solvation for PAN/solvents system revealed that 1) interaction of all solvents to polymer is strictly dependent on polymer stereoregularity, 2) each solvent have own favorable site in polymer ( $m m$ and $m r$ for aq $\mathrm{HNO}_{3}$, $m m r$ for DMSO, and basically $m r$ for DMF and DMAc), 3 ) the solvent power is related to the solvent ability to interact on the long meso $(m)$ sequence part in PAN, and 4) the observed solvation number of solvent was proved to be in good accordance with that theoretical solvation number. The tentative solvation form for each solvent is proposed by considering electro-magnetical influences on AN unit caused by interaction with solvents.

\section{REFERENCES}

1. H. F. Mark, S. M. Atlis, and E. Cerna, Ed., "Man-Made Fiber II," John Wiely \& Sons Inc., New York, N.Y., 1961.

2. K. Kamide, Y. Miyazaki, and H. Kobayasi, Polym. J., 14, 591 (1982).

3. K. Kamide, Y. Miyazaki, and H. Kobayasi, Polym. J., 17, 607 (1985)
4. K. Kamide, H. Yamazaki, and Y. Miyazaki, Polym. J., 18, 819 (1986).

5. H. Yamazaki, Y. Miyazaki, and K. Kamide, Polym. J., 23, 725 (1991).

6. K. Kamide, H. Yamazaki, K. Okajima, and K. Hikichi, Polym J., 17, 1233 (1985).

7. H. Yamazaki, Y. Miyazaki, and K. Kamide, Polym. J., 18, 1049 (1986).

8. K. Hisatani, K. Okajima, and K. Kamide, Polym. J., 28, 99 (1996).

9. K. Kamide, M. Saito, and K. Yasuda, Am. Chem. Soc., Symp. Ser., 489, 184 (1995).

10. K. Kamide, K. Okajima, and M. Saito, Polym. J., 13, 115(1981).

11. K. Kamide, S. Matsuda, and K. Kowsaka, Polym. J., 20, 231 (1988).

12. M. Hattori, M. Saito, K. Okajima, and K. Kamide, Polym. J., 27, 631 (1995)

13. H. Kobayasi, Kagaku, 23, 315 (1953).

14. S. Suzuki, Koubunshi, 11, 41 (1954).

15. S. Suzuki, Koubunshi, 11, 379 (1954).

16. H. Yamazaki, Doctorial Thesis, Kanazawa University, 1990.

17. H. Yamazaki, K. Okajima, and K. Kamide, Polym. J., 20, 1143 (1988).

18. A. Passynsky, J. Polym. Sci., 29, 61 (1958). 